\title{
Triterpenoids from Ganoderma lucidum inhibit the activation of EBV antigens as telomerase inhibitors
}

\author{
DONG-SHU ZHENG ${ }^{1}$ and LIANG-SHU CHEN ${ }^{2}$ \\ ${ }^{1}$ Department of Otolaryngology Head and Neck Surgery; ${ }^{2}$ Ward of Cadre Care, The First Affiliated Hospital \\ of Xiamen University, Xiamen, Fujian 361003, P.R. China \\ Received September 7, 2016; Accepted May 15, 2017
}

DOI: $10.3892 /$ etm.2017.4883

\begin{abstract}
Nasopharyngeal carcinoma (NPC) is a malignant disease that threatens the health of humans. To find effective agents for the inhibition of Epstein-Barr virus (EBV) infection, which is associated with NPC, a phytochemical investigation of Ganoderma lucidum was carried out in the present study. Five triterpenoids were identified, including ganoderic acid A (compound 1), ganoderic acid B (compound 2), ganoderol B (compound 3), ganodermanontriol (compound 4), and ganodermanondiol (compound 5), on the basis of spectroscopic analysis. An inhibition of EBV antigens activation assay was implemented to elucidate the triterpenoids from G. lucidum and potentially prevent NPC. All the triterpenoids showed significant inhibitory effects on both EBV EA and CA activation at $16 \mathrm{nmol}$. At $3.2 \mathrm{nmol}$, all the compounds moderately inhibited the activation of the two antigens. The activity of telomerase was inhibited by these triterpenoids at $10 \mu \mathrm{M}$. Molecular docking demonstrated that compound 1 was able to inhibit telomerase as a ligand. In addition, the physicochemical properties of these compounds were calculated to elucidate their drug-like properties. These results provided evidence for the application of these triterpenoids and whole G. lucidum in the treatment of NPC.
\end{abstract}

\section{Introduction}

Nasopharyngeal carcinoma (NPC) is a prevalent disease in southeastern Asia. In south China, it also threatens the health of humans; specifically, in the Cantonese region around Guangzhou, the incidence of NPC is $30-80$ per 100,000 individuals annually (1). Radiotherapy and chemotherapy can

Correspondence to: Dr Liang-Shu Chen, Ward of Cadre Care, The First Affiliated Hospital of Xiamen University, 55 Zhenhai Road, Xiamen, Fujian 361003, P.R. China

E-mail: chenliangshuxm@126.com

Key words: Ganoderma lucidum, triternepoids, nasopharyngeal carcinoma, Epstein-Barr virus antigens, telomerase inhibitor, physicochemical properties improve the prognosis of patients with NPC, but the survival period remains low due to drug resistance and metastasis (2). Genetic predisposition, Epstein-Barr virus (EBV) infection, and environmental conditions are major factors that lead to malignant transformation (3). Though the detailed etiology of NPC remains unclear, it has been demonstrated that EBV infection has a pivotal role in the pathogenesis of NPC through EBV-encoded latent genes $(4,5)$. Inhibiting EBV infection may represent a promising approach to preventing NPC (6).

Telomerase is a pivotal holoenzyme involved in the repair of damaged telomeres, which is the protective end of eukaryotic chromosomes, through reverse transcription (7). Cells infected by EBV will lead to telomere dysfunction, and the telomere length was increased by the active telomerase (8). Increased telomerase activity also directly affects EBV infection by facilitating potential EBV gene expression early in virus inoculation (9). Telomerase inhibition is a possible therapeutic strategy for the treatment of cancer (10).

Ganoderma lucidum (Leyss. ex Fr.) Karst is a mushroom distributed in the majority of areas in southern China. It is used as a healthy food and traditional medicine for treating insomnia, amnesia, fatigue and expectoration in Chinese folklore and has been included in the Pharmacopeia of the People's Republic of China (2015 Edition) (11). Previous phytochemical studies have elucidated that the major phytochemicals in G. lucidum are triterpenoids $(12,13)$, though steroids, fatty acids, nucleotides, nucleobases, lactones and alkaloids have also been reported (14-16). Pharmacological investigations on G. lucidum have revealed that it possesses various bioactivities, including anti-inflammation (17), anti-diabetes (18), neuroprotecion (19), and anti-cancer (20) properties. In addition, G. lucidum has been prescribed for the prevention of NPC in more than 100 hospitals, including ours. Therefore, the present study aimed to investigate the bioactive phytochemicals in G. lucidum that may be useful in the prevention of NPC. Herein, we report that triterpenoids from G. lucidum inhibit EBV antigens activation as telomerase inhibitors.

\section{Materials and methods}

Ethics statement. The study protocol was approved by the Ethical Committee of The First Hospital of Xiamen Univesity (Fujian, China) and written informed consent was obtained from all participants. 
Chemicals and reagents. Water was prepared from distilled water using a Milli-Q system (Millipore Laboratory, Bedford, MA, USA); 3-(4,5-dimethylthiazol-2-yl)-2,5-diphenyltetrazolium bromide (MTT), dimethyl sulfoxide (DMSO), and 12-o-tetradecanoylphorbol-13-acetate (TPA) were obtained from Sigma-Aldrich (Merck KGaA, Darmstadt, Germany). RPMI-1640 was supplied by Gibco (Thermo Fisher Scientific Inc., Rockville, MD, USA). A TRAP-polymerase chain reaction (PCR)-ELISA kit was purchased from Roche Diagnostics (Basel, Switzerland). The other solvents used in this study were of analytical purity grade. Sephadex LH-20 was purchased from GE Healthcare Life Sciences (Shanghai, China). Silica gel was obtained from Qingdao Oceanic Chemical Co., Ltd. (Qingdao, China).

Experimental materials. Fruit bodies of G. lucidum were purchased from Fujian Xianzhilou Biotechnology Co., Ltd. (Fujian, China) in 2013 and identified by Mr Feng Li at the same institute. A sample of fruiting bodies of G. lucidum was deposited in our institution as voucher specimen (M20130606). EBV early antigen (EA) positive serum and EBV capsid antigen (CA) positive serum were collected from 5 patients (male:femal, 2:3) with NPC at our institution between July and December 2015 according to the diagnostic guidelines from the Chinese Medical Association (21). Patients without positive EA or CA were excluded.

Extraction and isolation. Air dried fruit bodies of G. lucidum $(3.0 \mathrm{~kg})$ were ground and extracted with $95 \% \mathrm{EtOH}(6.0$ l) three times under reflux for $3 \mathrm{~h}$ each time. The solvent was evaporated under reduced pressure and the residue was suspended in water $(1.5 \mathrm{l})$ and partitioned successively with dichloromethane (DCM; 1.5 l) three times. Following this, the solvent was evaporated to yield the DCM extract $(65.0 \mathrm{~g})$.

DCM extract was subjected to common chromatography (CC) on silica gel eluted with gradient petroleum ether (PE)/ethyl acetate (EA) (from 100:0 to 10:90; v/v) and gave 7 fractions according to the thin-layer chromatography assay. Fraction 2 was separated on silica gel and crystalized in DCM to give compounds $3(18.5 \mathrm{mg})$ and $5(26.0 \mathrm{mg})$. Fraction 3 was subjected to CC over Sephadex LH-20 eluted with DCM to yield three subfractions. Subfraction 3 was chromatographed over silica gel with gradient PE/EA (from 100:0 to 50:50; v/v) to obtain compound $4(21.0 \mathrm{mg})$. Fraction 5 was handled with silica gel CC eluted with gradient PE/acetone and DCM/EA repeatedly to afford compounds $1(22.0 \mathrm{mg})$ and $2(10.5 \mathrm{mg})$. Fraction 6 was separated on silica gel CC with gradient DCM/acetone (from 100:0 to 70:30; v/v) and further purified by Sephadex LH-20 CC with isocratic DCM/MeOH (3:1; v/v) to yield compound $1(27.0 \mathrm{mg})$.

The compounds obtained were dissolved in $\mathrm{CDCl}_{3}$ and the nuclear magnetic resonance spectra were recorded on a Bruker DRX400 NMR spectrometer (Bruker, Billerica, MA, USA). Their chemical structures were identified on the basis of the spectra analysis.

Cell culture. $\mathrm{B}_{95-8}$ cells and Raji cells were purchased from Cell Bank of Shanghai Institute of Biological Sciences (Shanghai, China). Human nasopharyngeal carcinoma 5-8 F cells (NPC 5-8 F cells) were obtained from Nanjing Haeckel
Biotechnology Co., Ltd. (Nanjing, China). Cells were cultured in RPMI-1640 medium supplemented with $10 \%$ fetal bovine serum (FBS; Thermo Fisher Scientific, Inc.), $100 \mathrm{U} / \mathrm{ml}$ penicillin and $100 \mathrm{U} / \mathrm{ml}$ streptomycin, and incubated in a humid atmosphere containing $5 \% \mathrm{CO}_{2}$ at $37^{\circ} \mathrm{C}$.

MTT assay. To determine if there are toxic effects induced by natural compounds on Raji cells and B95-8 cells as well as the inhibitory effect on NPC 5-8 F cells, an MTT assay was performed. Cells in the flask were adjusted to a density of $1 \times 10^{6} / \mathrm{ml}$ and $100 \mu \mathrm{l}$ suspension was added into 96-well microplates for each well. Following this, the cells were divided into control group and test groups. Test groups were administered with compounds at a concentration of $1 \mathrm{mM}$. Following incubation for $48 \mathrm{~h}$, MTT was added into each well at $0.5 \mathrm{mg} / \mathrm{ml}$ further incubated for $4 \mathrm{~h}$ at $37^{\circ} \mathrm{C}$. The medium was subsequently removed and DMSO was used to dissolve the formazan. After $10 \mathrm{~min}$, the absorbance was measured at $550 \mathrm{~nm}$ on a microplate reader. Cell viability was expressed as a relative percentage of optical density (OD) values compared with the control group.

Inhibiton of EBV EA activation. Inhibiton of EBV EA activation was measured as previously described $(22,23)$. Raji cells, the EBV genome carrying lymphoblastoid cells derived from Burkitt's lymphoma, were adjusted to $1 \times 10^{6} / \mathrm{ml}$ and cultured in $1 \mathrm{ml}$ RPMI- 1640 for $48 \mathrm{~h}$ at $37^{\circ} \mathrm{C}$, with the medium containing $4 \mu \mathrm{mol} n$-butyric acid and 32 pmol TPA as co-inducers, as well as 16 or $3.2 \mathrm{nmol}$ compounds in DMSO. Smears were prepared from the cell suspension. Raji cells expressing EBV EA were stained with EBV EA-positive serum from patients with NPC and detected through an indirect immunofluorescence technique (24). The number of stained cells (positive cells) among 500 cells was recorded. Each assay was carried out three times. Inhibition of EBV EA activation was represented as a relative ratio compared with the positive control group (100\%), which was exposed to $4 \mu \mathrm{mol} n$-butyric acid and 32 pmol TPA. In the experiments, the ratio of EBV EA activation was typically $\sim 35 \%$.

Inhibiton of EBV CA activation. To further evaluate the inhibitory effects of compounds obtained on EBV antigens, $\mathrm{B}_{95-8}$ cells, the EBV-transformed tamarin cells were employed. According to a previously published protocol (22), the experimental procedure is similar to the assay above. Cells were diluted to $1 \times 10^{6} / \mathrm{ml}$ and cultivated in $1 \mathrm{ml}$ medium containing $4 \mu \mathrm{mol} n$-butyric acid and 16 or $3.2 \mathrm{nmol}$ of the compounds obtained. Following incubation at $37^{\circ} \mathrm{C}$ for $48 \mathrm{~h}$, smears were made and the cells were stained with EBV CA positive serum from patients with NPC. In total, 500 cells were counted and the number of stained cells was recorded. The inhibitory effect on EBV CA activation was expressed as a relative ratio compared with the positive control group (100\%) treated with $4 \mu \mathrm{mol} n$-butyric acid. The ratio of EBV CA activation was $\sim 30 \%$.

Telomerase inhibition assay. The inhibitory effects of these compounds on telomerase were determined based on the telomeric repeat amplification protocol (TRAP) (25). Herein, the TRAP-PCR-ELISA kit was employed according to the 
manufacturer's instructions, as previously described (26). Briefly, the NPC 5-6 F cells $\left(5 \times 10^{4}\right.$ cells $\left./ \mathrm{ml}\right)$ in logarithmic growth were incubated with the compounds at $10 \mu \mathrm{M}$ for $24 \mathrm{~h}$. Subsequently, cells were washed with PBS and lysed with lysis buffer. Subsequently, the lysate was centrifuged at $15,000 \mathrm{x} \mathrm{g}$ for $20 \mathrm{~min}$ at $4^{\circ} \mathrm{C}$ to isolate the supernatant for analysis. In total, $2 \mu$ l cellular extract was added into the reaction mixture. PCR was performed at $94^{\circ} \mathrm{C}$ for $120 \mathrm{sec}$ for initial denaturation, followed by 35 cycles of $94^{\circ} \mathrm{C}$ for $30 \mathrm{sec}, 50^{\circ} \mathrm{C}$ for $30 \mathrm{sec}$, and $72^{\circ} \mathrm{C}$ for $90 \mathrm{sec}$. Subsequently, PCR products $(20 \mu \mathrm{l})$ were hybridized with a digoxigenin-labeled telomeric repeat specific detection probe (Roche Diagnostics). PCR products were immobilized to a streptavidin-coated microplate via a biotin-labeled primer (5'-biotin-ACGACGTCCATAAGCA ACT-3'; Roche Diagnostics). Immobilized DNA fragments were detected with a peroxidase-conjugated anti-digoxigenin antibody (cat. no. 1185466; 1:1,000; Roche Diagnostics) and visualized in the presence of the stop regent. The absorbance was recorded on a microplate reader at $490 \mathrm{~nm}$. Results were presented as the inhibition ratio compared with the control group.

Molecular modeling. According to the results of telomerase inhibition, compound 1, a typical triterpenoid in G. lucidum, was chosen as the ligand to reveal the binding mode with telomerase through molecular docking. The 3D structure of compound 1 was generated by SYBYL sketch software X2.0 (Tripos, Inc., St. Louis, MO, USA). The protein crystal structure was retrieved from Protein Data Bank (http://www. rcsb.org/pdb) (PDB code, 3DU6); hydrogen atoms and charges were added, and water molecules were removed. The docking process was implemented using Surflex-Dock software X2.0 (Tripos, Inc.). In total, 20 ligand conformations were obtained.

Physicochemical properties of the compounds. Lipophilicity/hydrophilicity has a strong influence on the ADME properties of drugs in vivo. Hydrophobic drugs are inclined to bind to hydrophobic sites while hydrophilic ones favor hydrophilic positions. Hence, the lipophilicity/hydrophilicity expressed as $\log p$ was theoretically calculated to elucidate the physicochemical properties of these compounds. Typically, the values of $\log p$ for drugs were between 2 and 5 , which can be considered to indicate that the drugs are easily delivered to the binding sites (27).

Polar surface area (PSA) is defined as the sum of the surface over all polar atoms, primarily oxygen and nitrogen atoms, and their attached hydrogen atoms. PSA is typically used to optimize the drug's ability to permeate cells (28). Molecules with a PSA of $>140$ angstroms $^{2}$ tend to be poor at permeating cell membranes.

Statistical analysis. All data were expressed as the mean \pm standard deviation. GraphPad Prism 5.0 software (GraphPad Software, Inc., La Jolla, CA, USA) was employed for data analyses. Statistical differences of the data were analyzed by one way analysis of variance followed by Dunnett's test for multiple comparisons, and Student's t-test was employed for single comparisons. $\mathrm{P}<0.05$ was considered to indicate a statistically significant difference.
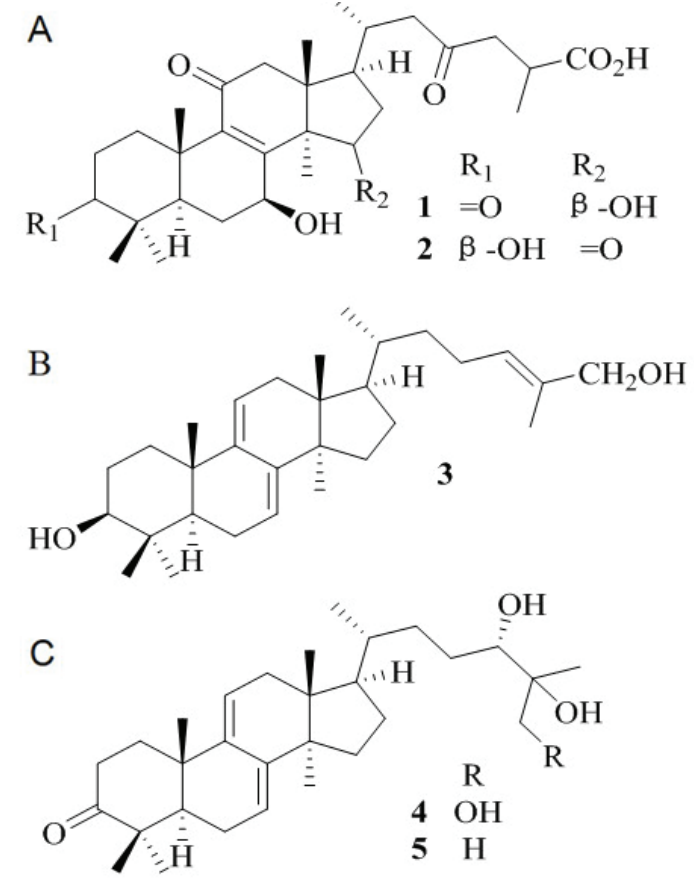

Figure 1. Structures of triterpenoids from Ganoderma lucidum extract. (A) Ganoderic acid A (compound 1) and ganoderic acid B (compound 2); (B) ganoderol B (compound 3); (C) ganodermanontriol (compound 4) and ganodermanondiol (compound 5).

\section{Results}

Phytochemical investigation. Phytochemical studies on the DCM extract of G. lucidum led to the isolation of five natural compounds. All the compounds showed positive results via the Liebermann-Burchard reaction. Their structures were identified as ganoderic acid A (compound 1), ganoderic acid B (compound 2), ganoderol B (compound 3), ganodermanontriol (compound 4), and ganodermanondiol (compound 5) (Fig. 1) on the analysis of nuclear magnetic resonance spectra and comparison of the data with existing literature (29-31).

Cells viability. To evaluate whether these triterpenoids had toxic effects on Raji cells, $\mathrm{B}_{95-8}$ cells and NPC 5-8 F cells. MTT assay was employed. The results demonstrated that the triterpenoids did not affect the survival of these cell lines and had no toxic effects against Raji cells and $\mathrm{B}_{95-8}$ cells, even when treated with a concentration of $1 \mathrm{mM}$ (Fig. 2). These results indicated the inhibitory effects of these compounds on EBV EA and CA activation should be further investigated. Conversely, the viability of NPC 5-8 F cells was significantly reduced by all five triterpenoids at $1 \mathrm{mM}$. The minimum viability was $81.87 \pm 5.10 \%$ after treatment with compound 1 , and the maximum viability was $89.67 \pm 2.12 \%$ induced by compound 5 .

Inhibiton of EBV EA and CA activation. The inhibitory effects of triterpenoids on EBV EA and CA activation were assessed via an indirect immunofluorescence assay. The results indicated that all five compounds contributed to the inhibition of EBV EA and CA activation (Fig. 3). When 


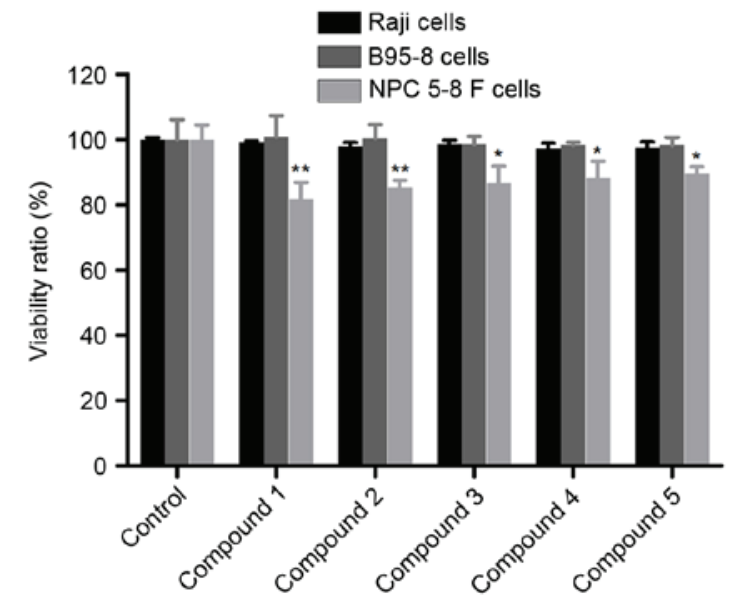

Figure 2. Effect of triterpenoids from Ganoderma lucidum on the viability of Raji cells, $B_{95-8}$ cells and NPC 5-8 F cells $(n=3)$. $P<0.05$ and ${ }^{* *} P<0.01$ vs. the control group. NPC, nasopharyngeal carcinoma.

A

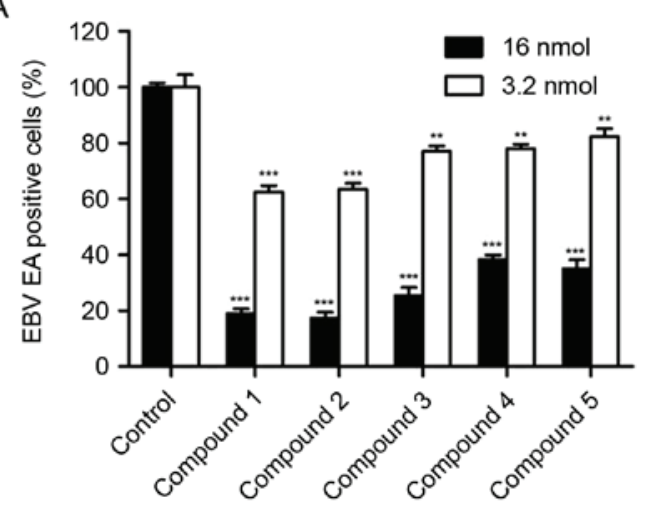

B

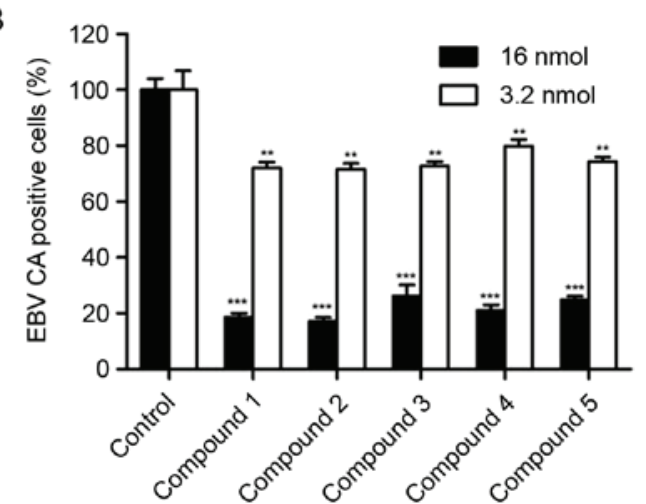

Figure 3. Inhibitory effects of triterpenoids from Ganoderma lucidum on EBV (A) EA and (B) CA activation ( $\mathrm{n}=3) .{ }^{* *} \mathrm{P}<0.01$ and ${ }^{* * * *} \mathrm{P}<0.001$ vs. the control group. EBV, Epstein-Barr virus; EA, early antigen; CA, capsid antigen.

administered at a high concentration $(16 \mathrm{nmol})$, compounds 1 and 2 exhibited the most potent inhibitory effects on EBV EA activation (18.97 \pm 1.76 and $17.40 \pm 2.10 \%)$, as compared with the others; all compounds were able to significantly inhibit the activation of EBV CA. At the low concentration of $3.2 \mathrm{nmol}$, all the compounds exhibited significant, yet moderate, inhibitory effects on EBV EA and CA. Of all the compounds, compounds 1 and 2 exhibited slightly greater inhibitiory

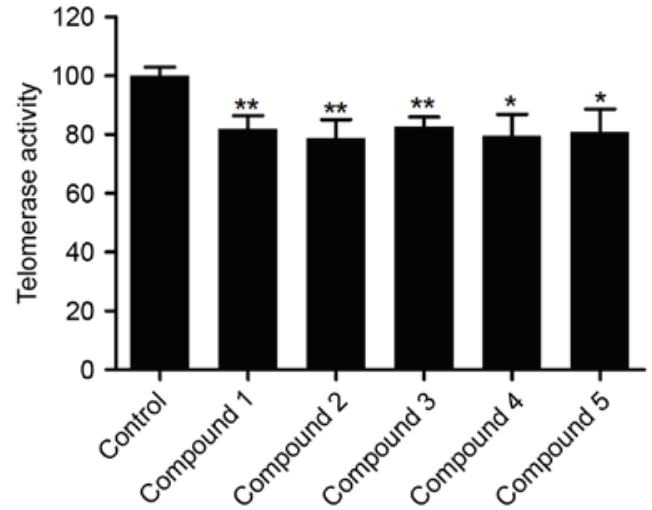

Figure 4. Relative inhibition ratio of telomerase by the triterpenoids from Ganoderma lucidum ( $\mathrm{n}=3)$. ${ }^{*} \mathrm{P}<0.05$ and $^{* *} \mathrm{P}<0.01$ vs. the control group.

effects $(62.43 \pm 2.30$ and $63.53 \pm 2.11 \%)$ than the others three compounds at $3.2 \mathrm{nmol}$.

Telomerase inhibition. Activity of telomerase in NPC 5-8 F cells was detected via the TRAP-PCR-ELISA method. The triterpenoids obtained exhibited significant inhibitory effects on telomerase. The relative inhibition ratio ranged from $78.62 \pm 6.41$ to $82.79 \pm 3.12 \%$ (Fig. 4). There was no notable difference in the inhibitory effects among these compounds.

Molecular docking. Molecular docking provides additional information about the interaction of compound 1 with telomerase. As shown in Fig. 5, the entire molecule of compound 1 is able to completely enter the binding pocket. The privileged conformation of compound 1 can bind to amino acid residues through hydrogen bonds, van der Waals force, electrostatic interaction and hydrophobic interaction. The 7-hydroxyl group of compound 1 forms a hydrogen bond with ASN-142 $(2.2 \AA)$ as a hydrogen donor. At the same time, 15-hydroxyl, 23-carbonyl and 26-carboxyl groups interacts as hydrogen acceptors with ASN-446 (1.9 ̊) and LYS-416 (2.1 and $2.1 \AA)$ to form hydrogen bonds (Fig. 5A). Compound 1 also interacts with LYS-406 and LYS-416 through electrostatic interaction. LEU-141, LEU-404, PHE-443 and ILE-444 contribute to the hydrophobic interaction. Meanwhile, van der Waals forces exist between compound 1 and several residues, including ASN-142, GLY-143, GLY-391, THR-403, TYR-405, LYS-406, LYS-416, CYS-445, ASN-446, SER-447 and CYS-473 (Fig. 5B). The total score was 6.67 , which suggests that there is moderate interaction between the ligand and telomerase. The docking results indicate that compound 1 is able to inhibit telomerase as a ligand.

Physicochemical properties of the triterpenoids. As shown in Table I, the $\log p$ values for the identified compounds 1 and 2 fall in the range of 2 to 5 , which indicates that they shall be well delivered to the binding sites. Compounds 3-5 exhibited higher $\log p$ values when compared with compounds 1 and 2 . On the contrary, the trend for PSA was the opposite; compounds 3-5 exhibited better permeability than compounds 1 and 2, which may attributed to the carboxyl groups of the two compounds. The oxygen atoms of carboxyl groups increase the PSA through the elevation of polarity. 
Table I. Physicochemical properties of the triterpenoids.

\begin{tabular}{lllr}
\hline Compounds & MW & $\log p$ & PSA \\
\hline 1 & 516.7 & 3.45 & 261.7 \\
2 & 516.7 & 2.79 & 214.4 \\
3 & 440.7 & 6.79 & 85.6 \\
4 & 498.8 & 5.59 & 125.8 \\
5 & 456.7 & 6.45 & 91.0 \\
\hline
\end{tabular}

MW, molecular weight; PSA, polar surface area.

\section{Discussion}

As a medicinal and edible fungus, G. lucidum has been used promote to health, prolong life and prevent diseases for $>2,000$ years (32). The major phytochemicals in G. lucidum are triterpenoids (33), which have attracted research attention as potential anti-cancer agents (34). Herein, we identified five triterpenoids from G. lucidum and evaluated their effects on EBV EA and CA activation, as well as the inhibition of telomerase.

NPC is a common disease in various geographical areas, particularly in southern China. Although the pathogenesis of NPC is unclear, it is well-established that EBV infection contributes to the progression of NPC as a major factor (35). Inhibiting the activation of EBV antigens to block the infection has been employed to discover bioactive substances for the prevention of NPC $(36,37)$. In the present study, it was established that the main triterpenoids in G. lucidum did not affect the viability of Raji cells and $\mathrm{B}_{95-8}$ cells, which facilitated further investigation into the inhibitory effect of these compounds on EBV EA and CA activation. The findings indicated that these triterpenoids are able to significantly inhibit the activation of EBV EA and CA, which suggests they may be useful in the prevention of NPC.

Telomerase is an enzyme that maintains the length of telomere and the integrity of the chromosome in frequently dividing cells, but becomes dormant in the majority of somatic cells during adulthood (26). Overexpression of telomerase has been demonstrated in $85-90 \%$ of human tumor cells but not at all in the majority of human normal somatic cells (38). Therefore, inhibiting telomerase may be a promising therapeutic strategy for the treatment of different types of cancer (10). Meanwhile, the activity of telomerase is closely associated with EBV infection and NPC (39). The results of the present study demonstrated that the tripertenoids in G. lucidum are able to moderately affect the activity of telomerase and the survival of NPC 5-8 F cells, which further suggested that G. lucidum may be used in the prevention of NPC. In addition, the molecular docking findings complement the experimental results that the triterpenoids can moderately inhibit the activity of telomerase.

Physicochemical properties of compounds affect the application; therefore, the physicochemical properties of these triterpenoids, including $\log p$ and PSA, were calculated in the present study. These triterpenoids consist of four fused aliphatic rings together with a aliphatic chain, which provide superior lipophilicity. Compounds 1 and 2 possess a carboxyl

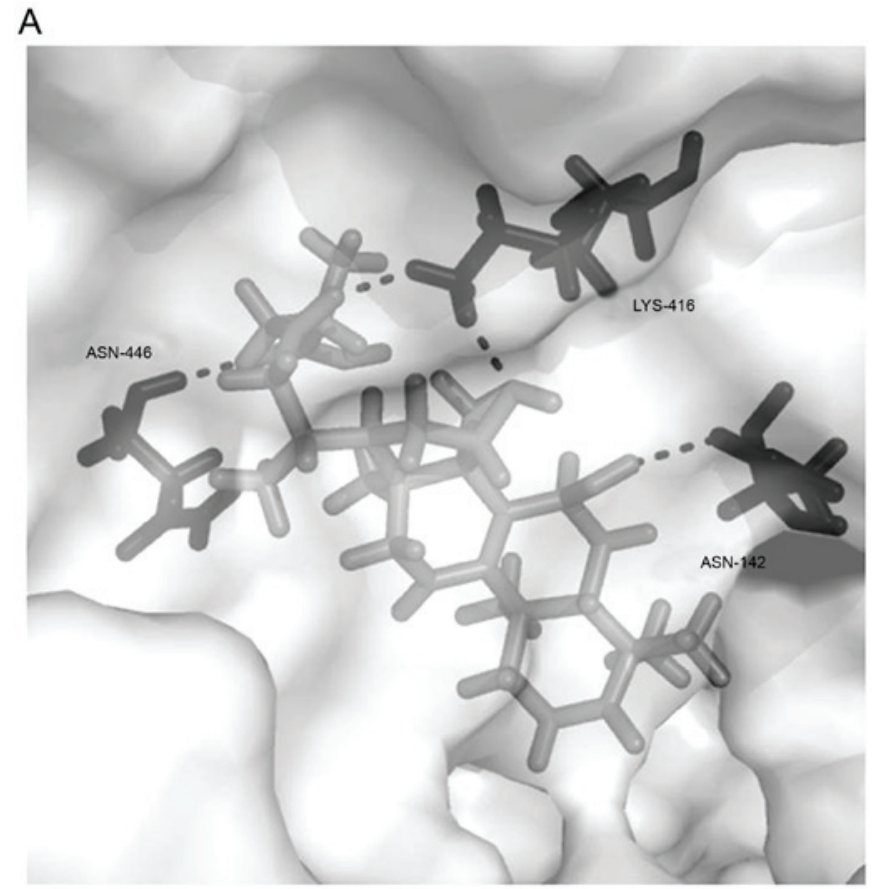

B

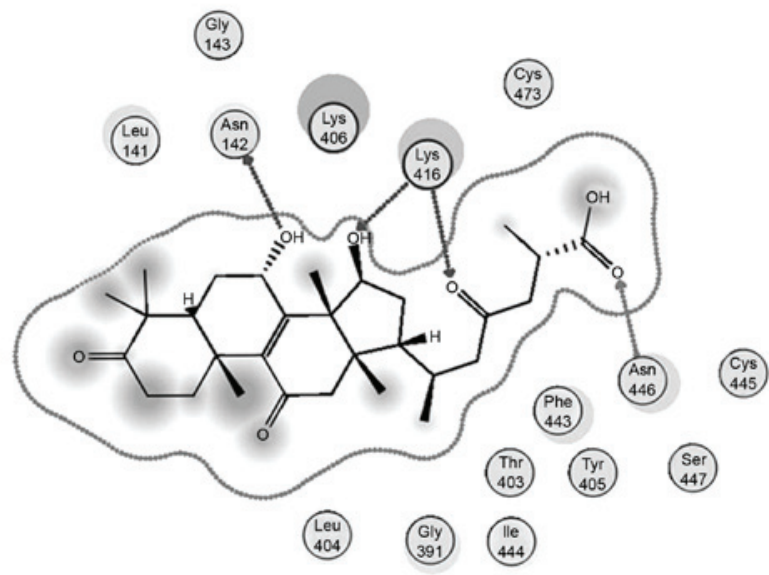

Figure 5. Binding model for compound 1 with telomerase. (A) Stereoview of compound 1 (gray) in the binding pocket interacting with amino acids residues (black) in the binding site via hydrogen bonds (black dashes). (B) Two-dimensional diagram of the interaction between compound 1 and telomerase.

group, which may positively contribute to the hydrophilicity and result in a suitable $\log p$ value. Furthermore, the oxygen atoms in carboxyl groups also increase the polarity of the whole molecule and give rise to a large PSA.

In the present study, five triterpenoids were identified as the major phytochemicals in G. lucidum. Pharmacological evaluation in vitro demonstrated that these triterpenoids were able to inhibit the activation of EBV antigens at 16 and $3.2 \mathrm{nmol}$ to different extents. The triterpenoids also inhibited the activity of telomerase, which is an enzyme associated with EBV infection. Molecular docking has further confirmed the inhibitory effect of compound 1 on telomerase. The physicochemical properties of the triterpenoids elucidated in the present study provide a primary outline of their drug-like properties. The results of the present study suggest that G. lucidum may be useful in the treatment of NPC. 


\section{Acknowledgments}

We would like to thank Dr Yang Wang at the University of Xiamen (Xiamen, China) for molecular simulation.

\section{References}

1. Liao K, Xia B, Zhuang QY, Hou MJ, Zhang YJ, Luo B, Qiu Y, Gao YF, Li XJ, Chen HF, et al: Parthenolide inhibits cancer stem-like side population of nasopharyngeal carcinoma cells via suppression of the NF- $\kappa \mathrm{B} / \mathrm{COX}-2$ pathway. Theranostics 5: 302-321, 2015.

2. Wei WI and Sham JS: Nasopharyngeal carcinoma. Lancet 365: 2041-2054, 2005.

3. Hildesheim A and Levine PH: Etiology of nasopharyngeal carcinoma: A review. Epidemiol Rev 15: 466-485, 1993.

4. Petersson F: Nasopharyngeal carcinoma: A review. Semin Diagn Pathol 32: 54-73, 2015.

5. Liu Q, Guan X, Lv J, Li X, Wang Y and Li L: Limb-bud and heart $(\mathrm{LBH})$ functions as a tumor suppressor of nasopharyngeal carcinoma by inducing G1/S cell cycle arrest. Sci Rep 5: 7626, 2015

6. Stoker SD, Novalić Z, Wildeman MA, Huitema AD, Verkuijlen SA, Juwana H, Greijer AE, Tan IB, Middeldorp JM and de Boer JP: Epstein-Barr virus-targeted therapy in nasopharyngeal carcinoma. J Cancer Res Clin Oncol 141: 1845-1857, 2015.

7. Bellon $\mathrm{M}$ and Nicot C: Regulation of telomerase and telomeres: Human tumor viruses take control. J Natl Cancer Inst 100 98-108, 2008.

8. Kamranvar SA, Chen X and Masucci MG: Telomere dysfunction and activation of alternative lengthening of telomeres in B-lymphocytes infected by Epstein-Barr virus. Oncogene 32: 5522-5530, 2013

9. Rac J, Haas F, Schumacher A, Middeldorp JM, Delecluse HJ, Speck RF, Bernasconi M and Nadal D: Telomerase activity impacts on Epstein-Barr virus infection of AGS cells. PLoS One 10: e0123645, 2015.

10. Feldser DM and Greider CW: Short telomeres limit tumor progression in vivo by inducing senescence. Cancer Cell 11: 461-469, 2007.

11. Chen Z, Shao M, Chen X, Yu W, Wu Z, Ding J, Yu D, Wang P, Wang J, Wang L, et al (eds): Ganoderma. In: Zhongguo Yao Dian. Vol 1. 2015 edition. China Medical Science Press, Beijing, pp188-189, 2015.

12. Liu DZ, Zhu YQ, Li XF, Shan WG and Gao PF: New triterpenoids from the fruiting bodies of Ganoderma lucidum and their bioactivities. Chem Biodivers 11: 982-986, 2014.

13. Luo Q, Wang XL, Di L, Yan YM, Lu Q, Yang XH, Hu DB and Cheng YX: Isolation and identification of renoprotective substances from the mushroom Ganoderma lucidum. Tetrahedron 71: 840-845, 2015.

14. Lv GP, Zhao J, Duan JA, Tang YP and Li SP: Comparison of sterols and fatty acids in two species of Ganoderma. Chem Cent J 6: 10, 2012.

15. Chen Y, Bicker W, Wu J, Xie M and Lindner W: Simultaneous determination of 16 nucleosides and nucleobases by hydrophilic interaction chromatography and its application to the quality evaluation of Ganoderma. J Agric Food Chem 60: 4243-4252, 2012.

16. Wang XF, Yan YM, Wang XL, Ma XJ, Fu XY and Cheng YX: Two new compounds from Ganoderma lucidum. J Asian Nat Prod Res 17: 329-332, 2015.

17. Hasnat MA, Pervin M, Cha KM, Kim SK and Lim BO: Anti-inflammatory activity on mice of extract of Ganoderma lucidum grown on rice via modulation of MAPK and NF-кB pathways. Phytochemistry 114: 125-136, 2015.

18. Pan D, Wang L, Chen C, Hu B and Zhou P: Isolation and characterization of a hyperbranched proteoglycan from Ganoderma lucidum for anti-diabetes. Carbohydr Polym 117: 106-114, 2015

19. Zhang W, Zhang Q, Deng W, Li Y, Xing G, Shi X and Du Y: Neuroprotective effect of pretreatment with Ganoderma lucidum in cerebral ischemia/reperfusion injury in rat hippocampus. Neural Regen Res 9: 1446-1452, 2014.
20. Dai S, Liu J, Sun X and Wang N: Ganoderma lucidum inhibit proliferation of human ovarian cancer cells by suppressing VEGF expression and up-regulating the expression of connexin 43 . BMC Complement Altern Med 14: 434, 2014.

21. Chinese Society of Radiation Oncology of Chinese Medical Association: Clinical diagnostic staging criteria of nasopharyngeal carcinoma. New Med 28: 672, 1997.

22. Tanaka R, Ishikawa $\mathrm{Y}$, Minami T, Minoura K, Tokuda $\mathrm{H}$ and Mateunaga S: Two new anti-tumor promoting serratane-type triterpenoids from the stem bark of Picea jezoensis var. jezoensis. Planta Med 69: 1041-1047, 2003.

23. Kapadia GJ, Azuine MA, Tokuda H, Hang E, Mukainaka T, Nishino $\mathrm{H}$ and Sridhar R: Inhibitory effect of herbal remedies on 12-O-tetradecanoylphorbol-13-acetate-promoted Epstein-Barr virus early antigen activation. Pharmacol Res 45: 213-220, 2002.

24. Murakami A, Ohgashi H and Koshimizu K: Anti-tumor promotion with food phytochemicals: A strategy for cancer chemoprevention. Biosci Biotechnol Biochem 60: 1-8, 1996.

25. Kim NW, Piatyszek MA, Prowse KR, Harley CB, West MD, Ho PL, Coviello GM, Wright WE, Weinrich SL and Shay JW: Specific association of human telomerase activity with immortal cells and cancer. Science 266: 2011-2015, 1994.

26. Zhang XM, Qiu M, Sun J, Zhang YB, Yang YS, Wang XL, Tang JF and Zhu HL: Synthesis, biological evaluation and molecular docking studies of 1,3,4-oxadiazole derivatives possessing 1,4-benzodioxan moiety as potential anticancer agents. Bioorg Med Chem 19: 6518-6524, 2011.

27. Damu GL, Cui SF, Peng XM, Wen QM, Cai GX and Zhou CH: Synthesis and bioactive evaluation of a novel series of coumarinazoles. Bioorg Med Chem Lett 24: 3605-3608, 2014

28. Caron G and Ermondi G: Molecular descriptors for polarity: The need for going beyond polar surface area. Future Med Chem, 2016.

29. Arisawa M, Fujita A, Morita N, Hayashi T, Shimizu M, Kikuchi T, Kadota $\mathrm{S}$ and Tezuka Y: Revision of ${ }^{1} \mathrm{H}$ - and ${ }^{13} \mathrm{C}-\mathrm{nmr}$ assignments of lanostanoids from Ganoderma lucidum by 2D-nmr studies. J Nat Prod 51: 54-59, 1988.

30. Fujita A, Arisawa M, Saga M, Hayashi T and Morita N: Two new lanostanoids from Ganoderma lucidum. J Nat Prod 49: $1122-1125,1986$.

31. Kubota T, Asaka Y, Miura I and Mori H: Structures of ganoderic acid $\mathrm{A}$ and $\mathrm{B}$, two new lanostane type bitter triterpenes from Ganoderma lucidum (FR.) KARST. Helv Chim Acta 65: 611-619, 1982.

32. Klupp NL, Kiat H, Bensoussan A, Steiner GZ and Chang DH: A double-blind, randomised, placebo-controlled trial of Ganoderma lucidum for the treatment of cardiovascular risk factors of metabolic syndrome. Sci Rep 6: 29540, 2016.

33. Shao Y, Qiao L, Wu L, Sun X, Zhu D, Yang G, Zhang X, Mao X, Chen W, Liang W, et al: Structure identification and anti-cancer pharmacological prediction of triterpenes from Ganoderma lucidum. Molecules 21: pii: E678, 2016.

34. Gill BS, Kumar S and Navgeet: Triterpenes in cancer: Significance and their influence. Mol Biol Rep 43: 881-896, 2016.

35. Song LB, Zeng MS, Liao WT, Zhang L, Mo HY, Liu WL, Shao JY, Wu QL, Li MZ, Xia YF, et al: Bmi-1 is a novel molecular marker of nasopharyngeal carcinoma progression and immortalizes primary human nasopharyngeal epithelial cells. Cancer Res 66: 6225-6232, 2006.

36. Manosroi A, Jantrawut P, Ogihara E, Yamamoto A, Fukatsu M, Yasukawa K, Tokuda H, Suzuki N, Manosroi J and Akihisa T: Biological activities of phenolic compounds and triterpenoids from the galls of Terminalia chebula. Chem Biodivers 10 1448-1463, 2013.

37. Liu S, Li H, Chen L, Yang L, Li L, Tao Y, Li W, Li Z, Liu H, Tang M, et al: (-)-Epigallocatechin-3-gallate inhibition of Epstein-Barr virus spontaneous lytic infection involves ERK1/2 and PI3-K/Akt signaling in EBV-positive cells. Carcinogenesis 34: 627-637, 2013.

38. Tauchi T, Shin-ya K, Sashida G, Sumi M, Okabe S, Ohyashiki JH and Ohyashiki K: Telomerase inhibition with a novel G-quadruplex-interactive agent, telomestatin: In vitro and in vivo studies in acute leukemia. Oncogene 25: 5719-5725, 2006.

39. Ding L, Li L, Yang J, Zhou S, Li W, Tang M, Shi Y, Yi W and Cao Y: Latent membrane protein 1 encoded by Epstein-Barr virus induces telomerase activity via $1^{1 \mathrm{NK} 4 \mathrm{~A}} / \mathrm{Rb} / \mathrm{E} 2 \mathrm{~F} 1$ and JNK signaling pathways. J Med Virol 79: 1153-1163, 2007. 